\title{
Recent Advances in the Analysis of Nitrogen by EPMA
}

\author{
Anette von der Handt ${ }^{1}$, J. Mosenfelder ${ }^{1}$, C. Dalou ${ }^{1}$, and M.M. Hirschmann ${ }^{1}$ \\ ${ }^{1}$ University of Minnesota, Dept. of Earth Sciences, Minneapolis (USA) \\ * Corresponding author: avdhandt@umn.edu
}

The analysis of ultra-light elements such as nitrogen by electron probe microanalysis (EPMA) has consistently been extremely challenging due to its low fluorescence yield, resulting in low count rates and poor peak-to-background ratios [1,2]. Accordingly, comparatively few studies have been carried out by EPMA on nitrogen in silicate glasses [c.f. 3] and minerals. Of those, even fewer studies report detailed information on analytical conditions, detection limits and uncertainties. One study [4] reports a practical detection limit of $1500 \mathrm{ppm}$, limiting its application to samples with higher nitrogen contents. Other studies achieve lower detection limits using a calibration curve approach - similar to what is standard protocol for $\mathrm{N}$ analysis in steels - but its application is then then either limited to simple systems or constrained by the availability of suitable standards.

We will show that by applying a rigorous analytical protocol for EPMA analysis, one can drastically improve detection limits and accuracy even in compositionally complex materials within a reasonable analysis time. For this study we chose a series of nitrogen-bearing silicate glasses and iron alloys that were synthesized over a wide range of oxygen fugacities ( $\Delta \mathrm{IW}-3.3$ to $\Delta \mathrm{IW}-0.5)$ and pressures $(1.2$ to $3 \mathrm{GPa})$ [3]. Nitrogen contents ranged from 0.04 to $5.42 \mathrm{wt} \%$ in the silicate glasses and 0.98 to $3.54 \mathrm{wt} \%$ in the iron alloys. Errors are usually better than $3 \%$ for concentrations $>0.6 \mathrm{wt} \%$.

Most importantly, the strong background curvature on LDE monochromators for nitrogen demands application of advanced exponential background models, fit to wavelength scans (Figure 1). The presence of higher order metal lines close to the $\mathrm{N}$ peak has to be considered when choosing background positions. Acquisition on multiple spectrometers simultaneously can drastically shorten analysis time and allows to monitor for surface artefacts that are detrimental to the accuracy of the data. Subsurface charging effects can also create significant artifacts in EPMA analysis of nitrogen.

In addition, recent innovations in electron microprobe technology include a novel type of soft-X-ray emission spectrometer (SXES) composed of a CCD area detector coupled to multiple varied-line-spacing (VLS) aberration-corrected gratings that cover a combined energy range from $50 \mathrm{eV}$ to $2.3 \mathrm{keV}$. It provides higher spectral resolution and higher sensitivity for soft X-rays $(<1 \mathrm{keV})$ relative to traditional wavelength dispersive spectrometers (WDS), yielding information on electronic structure and bonding [5].

We investigated our samples also by SXES with a JS200N and JS300N grating. Silicate glasses and iron alloys yielded significantly different peak distributions of the second order N-K lines (Figure 2). Peak positions in iron alloys are shifted toward lower energies by $1 \mathrm{eV}$ compared to the silicate glasses. There are no systematic peak shifts with changes in pressure or oxygen fugacity in either the glass or alloy spectra. Although Ti contents are insignificant in both phases, the pathological interference with the second order Ti Ll-line can be well resolved through SXES, in contrast to WDS.

With modern EPMA instrumentation and careful protocols, high quality analysis of nitrogen is possible and can be incorporated in routine analysis protocols. 
References:

[1] M Raudsepp, Canadian Mineralogist 33 (1995), pp. 203-203.

[2] GF Bastin, HJM Heijligers, Scanning 13 (1991), pp. 325-342.

[3] C Dalou, M Hirschmann, A v.d.Handt, J Mosenfelder, and L Armstrong, EPSL 458 (2017), pp. 141.

[4] M Roskosz et al. Geochimica et Cosmochimica Acta, 121 (2013), pp. 15-28.

[5] M. Terauchi, In: TEM Characterization of Nanomaterials (Springer: Berlin, Heidelberg), p.287-331.

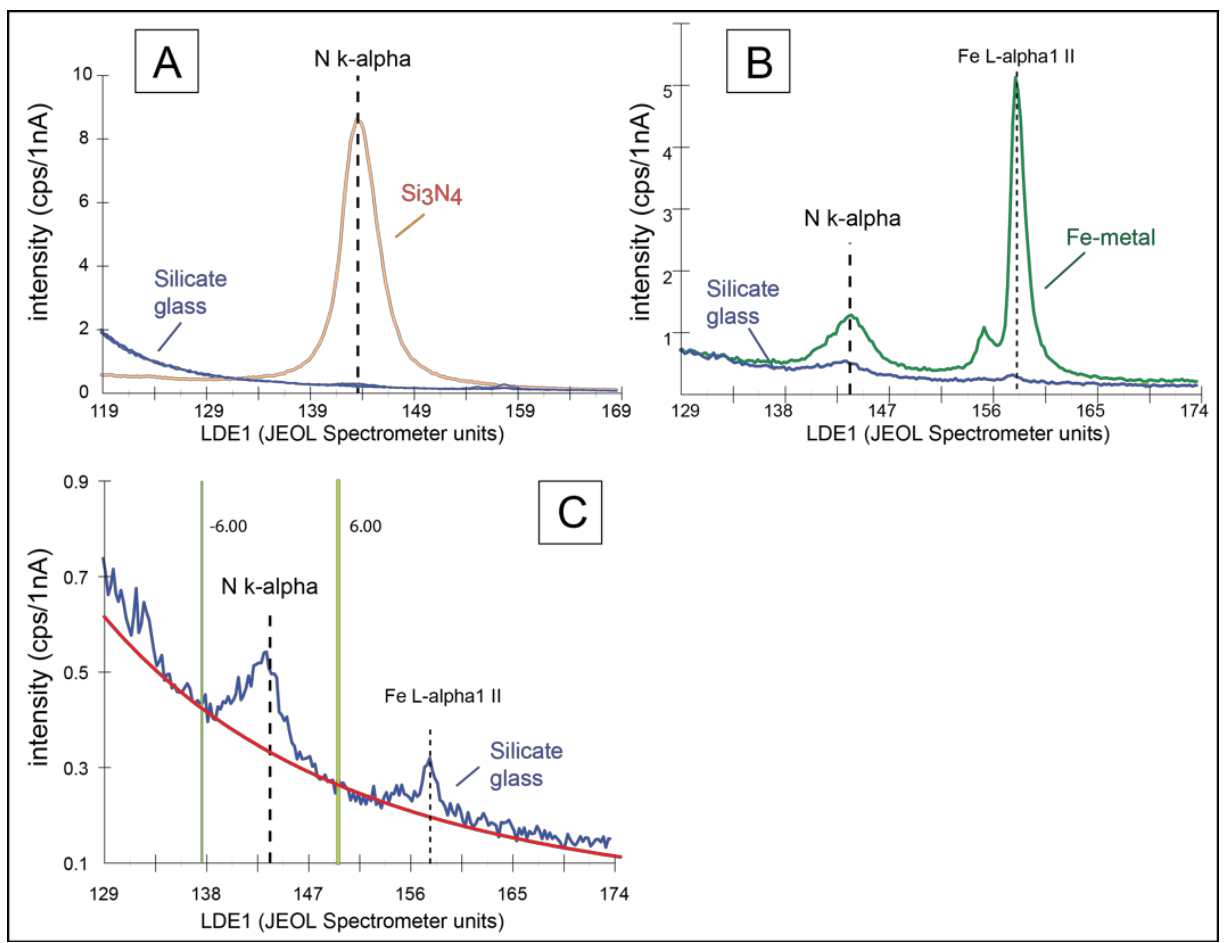

Figure 1. Nitrogen spectra recorded on LDE1 on various materials. A: Comparison of Si3N 4 and Nbearing silicate glasses highlighting the different background geometry. B: Position of higher order metal lines close to the $\mathrm{N} \mathrm{k}$-alpha peak such as Fe needs to be carefully recorded. C: Highly curved background

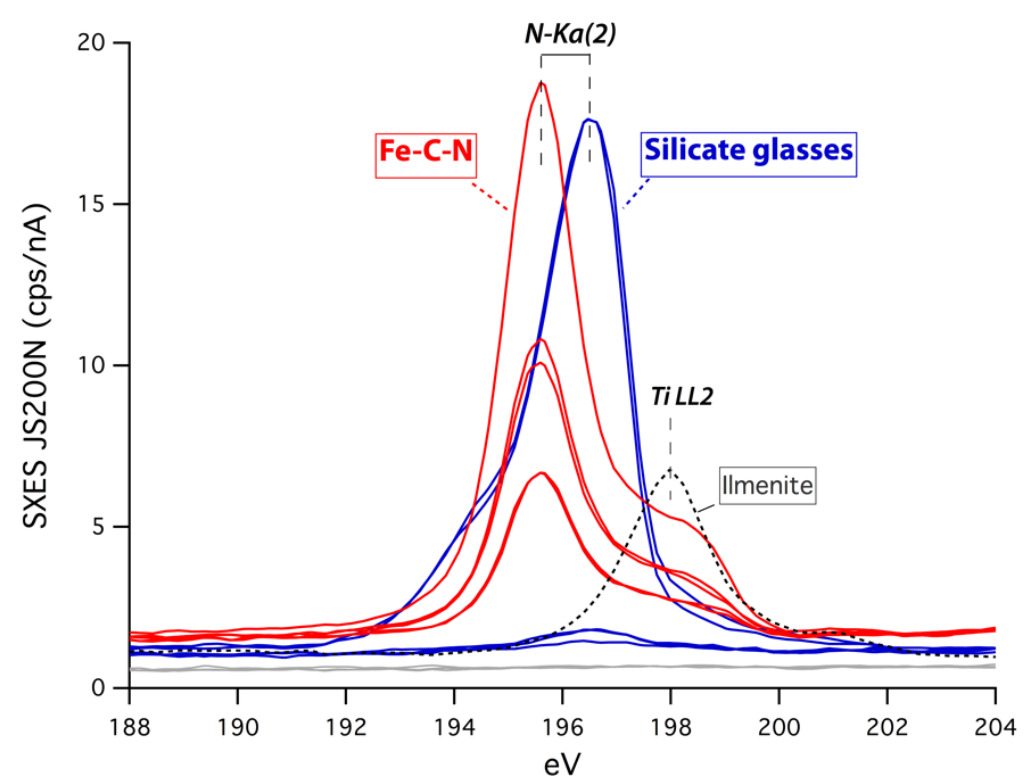

geometry necessitates exponential background fits in order to obtain accurate results by EPMA.

Figure 2. Comparison of soft X-ray spectra from nitrogen-bearing silicate glasses and iron alloys analyzed with the JS300N grating $(5 \mathrm{kV}, 100 \mathrm{n})$. A significant peak shift between silicate glasses and alloys can be observed as well as satellite bands either on the high-energy side in the alloys from conduction bands or satellite bands on the low-energy side in the glasses from valence bands. 